TRANSACTIONS OF THE

AMERICAN MATHEMATICAL SOCIETY

Volume 353, Number 10, Pages 4219-4234

S 0002-9947(01)02812-4

Article electronically published on June 6, 2001

\title{
ON THE RELATION BETWEEN UPPER CENTRAL QUOTIENTS AND LOWER CENTRAL SERIES OF A GROUP
}

\author{
GRAHAM ELLIS
}

\begin{abstract}
Let $H$ be a group with a normal subgroup $N$ contained in the upper central subgroup $Z_{c} H$. In this article we study the influence of the quotient group $G=H / N$ on the lower central subgroup $\gamma_{c+1} H$. In particular, for any finite group $G$ we give bounds on the order and exponent of $\gamma_{c+1} H$. For $G$ equal to a dihedral group, or quaternion group, or extra-special group we list all possible groups that can arise as $\gamma_{c+1} H$. Our proofs involve: (i) the Baer invariants of $G$, (ii) the Schur multiplier $\mathcal{M}(L, G)$ of $G$ relative to a normal subgroup $L$, and (iii) the nonabelian tensor product of groups. Some results on the nonabelian tensor product may be of independent interest.
\end{abstract}

\section{INTRODUCTION}

A group $H$ gives rise to an upper central series $1=Z_{0} H \leq Z_{1} H \leq \cdots$ and a lower central series $H=\gamma_{1} H \geq \gamma_{2} H \geq \cdots$. In this article we consider a normal subgroup $N \unlhd H$ contained in $Z_{c} H$, and study the influence of the quotient $G=H / N$ on the lower central group $\gamma_{c+1} H$. An old result of R. Baer [1] 18] states that $\gamma_{c+1} H$ is finite whenever the quotient $G$ is finite. We develop Baer's techniques to obtain the following three results.

A. For any finite $G$ we give an upper bound on the order of $\gamma_{c+1} H$. (A previous paper 9] gives a bound on $\left|\gamma_{c+1} H\right|$ when $G$ is finite nilpotent; the present result incorporates a small improvement in this case. Several authors have given bounds when $c=1$. In particular, there are papers by J.A. Green [17], J. Wiegold [29], [30], W. Gaschütz et al. [15], and M.R. Jones [19, [20, [21]. The case $c=1$ is also studied in [11] where the results are slightly sharper than those obtained by specialising our general bound to $c=1$.)

B. For any finite $G$ we give an upper bound on the exponent of $\gamma_{c+1} H$. (For $c=1$ this provides a generalisation of a result of A. Lubotzky and A. Mann [23] on the exponent of the Schur multiplier $\mathcal{M}(G)$ of a powerful $p$-group $G$; it also sharpens a bound of M.R. Jones [21] on the exponent of the Schur multiplier of a prime-power group. Furthermore, for $c \geq 1$ our bound yields a generalisation and sharpening of an estimate, given in [7], on the exponent of the $c$-nilpotent Baer invariant $M^{(c)}(G)$. This improvement for $c \geq 1$ has the following practical implication. An electronically down-loadable appendix to the paper [12 contains a MAGMA computer program for calculating a number of homotopy-theoretic constructions. In particular, it contains a function for computing $M^{(c)}(G)$ which requires, as input data, a finite presentation of a finite group $G$ together with any positive integer $q$

Received by the editors February 12, 1999.

2000 Mathematics Subject Classification. Primary 20F14, $20 \mathrm{~F} 12$.

(C)2001 American Mathematical Society 
divisible by $e^{c}$ where $e$ denotes the exponent of $M^{(c)}(G)$. The improved estimate for $e$ helps in choosing a suitable value for $q$.)

C. For $G$ equal to a dihedral group, or quaternion group, or extra-special group we list all possible groups that can arise as $\gamma_{c+1} H$. (This extends the work of N.D. Gupta and M.R.R. Moghaddam [16] which handles the dihedral 2-groups. It also extends the work of D. MacHale and P.Ó’Murchú [26], and J. Burns et al. [8] which treats all groups $G$ of order at most 30 for $c=1$, and all groups $G$ of order at most 16 for $c=2$.)

A precise statement of results A-C is provided in Section 2. Their proofs are given in Sections 4-6 respectively. The proofs involve three techniques with which the reader may not be too familiar. The first is the use of a nonabelian tensor product of groups. The second is the use of a Schur multiplier $\mathcal{M}(N, G)$ of a group $G$ relative to a normal subgroup $N$. The third is the use of Baer invariants of a group. Relevant details of these techniques are recalled, and developed, in Section 3. Some results in Section 3 (in particular Propositions 5,8 and 9) may be of independent interest.

\section{Statement of Results}

Let a group $G$ be presented as the quotient of a free group $F$ by a normal subgroup $R$. We state our results in terms of the Baer invariants

$$
M^{(c)}(G)=\frac{R \cap \gamma_{c+1} F}{\gamma_{c+1}(R, F)}, \quad c \geq 1,
$$

and related invariants

$$
\gamma_{c+1}^{*}(G)=\frac{\gamma_{c+1} F}{\gamma_{c+1}(R, F)}
$$

of the group $G$, where $\gamma_{1}(R, F)=R, \gamma_{c+1}(R, F)=\left[\gamma_{c}(R, F), F\right]$ and $\gamma_{c+1} F=$ $\gamma_{c+1}(F, F)$. It was shown by R. Baer [1] (see also [14] [25]) that these invariants are, up to group isomorphism, independent of the choice of free presentation of $G$. Note that there are canonical actions of $G$ on $M^{(c)}(G)$ and $\gamma_{c+1}^{*}(G)$ given by conjugation in $F / \gamma_{c+1}(R, F)$.

If $G$ is of the form $G \cong H / N$ with $N$ a normal subgroup of $H$ contained in $Z_{c} H$, then it is routine [6] to establish the existence of the canonical short exact sequence

$$
A \longmapsto \gamma_{c+1}^{*}(G) \longrightarrow \gamma_{c+1} H
$$

where $A$ is a submodule of the $\mathbf{Z} G$-module $M^{(c)}(G)$. We thus have inequalities (in which $\leq$ can be taken to mean 'divides')

$$
\begin{gathered}
\left|\gamma_{c+1} H\right| \leq\left|\gamma_{c+1}^{*}(G)\right|=\left|M^{(c)}(G)\right|\left|\gamma_{c+1} G\right| \\
\exp \left(\gamma_{c+1} H\right) \leq \exp \left(\gamma_{c+1}^{*}(G)\right)
\end{gathered}
$$

involving the orders and exponents of groups. Since $M^{(c)}(G)$ is a subgroup of $\gamma_{c+1}^{*}(G)$, we also have an inequality

$$
\exp \left(M^{(c)}(G)\right) \leq \exp \left(\gamma_{c+1}^{*}(G)\right) .
$$

Furthermore, a group $K$ arises as $\gamma_{c+1} H$ for some $H$ if and only if

$$
K \cong \frac{\gamma_{c+1}^{*}(G)}{A}
$$

with $A$ a submodule of $M^{(c)}(G)$. 
Observations (1), (2) and (4) allow us to state results A-C in terms of the invariant $\gamma_{c+1}^{*}(G)$. For the statement of result $\mathrm{A}$ we let $\chi_{c}(d)$ denote the number of elements in a basis of the free abelian group $\gamma_{c} F / \gamma_{c+1} F$ with $F$ the free group on $d$ generators. (There is a well-known formula for $\chi_{c}(d)$ due to E. Witt [27]. Let $\mu(m)$ be the Möbius function, defined for all positive integers by $\mu(1)=1, \mu(p)=-1$ if $p$ is a prime, $\mu\left(p^{k}\right)=0$ for $k>1$, and $\mu(a b)=\mu(a) \mu(b)$ if $a$ and $b$ are coprime integers. Witt's formula is

$$
\chi_{c}(d)=(1 / c) \sum_{m \mid c} \mu(m) d^{(c / m)}
$$

where $m$ runs through all divisors of $c$. Thus, for instance, $\chi_{2}(d)=\left(d^{2}-d\right) / 2$, $\chi_{3}(d)=\left(d^{3}-d\right) / 3, \chi_{4}(d)=\left(d^{4}-d^{2}\right) / 4$. $)$

For an arbitrary finite abelian $p$-group $A$ we define the integer

$$
\Lambda_{c}(A)=e_{1} \chi_{c+1}\left(d_{1}\right)+\sum_{j=2}^{k} e_{j}\left\{\chi_{c+1}\left(d_{1}+\cdots+d_{j}\right)-\chi_{c+1}\left(d_{1}+\cdots+d_{j-1}\right)\right\}
$$

where the parameters $d_{j}, e_{j}, k$ are determined by expressing $A$ uniquely in the form

$$
A \cong\left(C_{p^{e_{1}}}\right)^{d_{1}} \times\left(C_{p^{e_{2}}}\right)^{d_{2}} \times \cdots \times\left(C_{p^{e_{k}}}\right)^{d_{k}}
$$

with $e_{1}>e_{2}>\cdots>e_{k} \geq 1$.

For an arbitrary finite $d$-generator $p$-group $P$ we define the integer

$$
\Psi_{c}(P)=m_{c+1} d+m_{c} d^{2}+\cdots+m_{2} d^{c}
$$

where the terms of the lower central series of $P$ have orders $\left|\gamma_{j}(P)\right|=p^{m_{j}}$.

Note that an arbitrary finite group $G$ has a smallest term $L$ in its lower central series, namely the unique group $L=\gamma_{r} G$ that satisfies $\gamma_{r} G=\gamma_{r+1} G$. Suppose that $P$ is a $d$-generator $p$-Sylow subgroup of $G$ with Frattini subgroup $\Phi(P)=[P, P] P^{p}$, that $P /(P \cap[G, G])$ is a $\delta$-generator group, that $(L \cap P) /(L \cap \Phi(P))$ has order $p^{t}$, that $L \cap P$ has order $p^{\beta}$, and that $[L \cap P, P]$ has order $p^{\beta^{\prime}}$. We use these various parameters to define the integer

$$
\Theta_{c}(G, L, P)=\beta+\left(\omega-\beta^{\prime}\right)\left(1+\delta+\delta^{2}+\cdots+\delta^{c-1}\right),
$$

where

$$
\omega=d \beta-(1 / 2) t(t+1)
$$

Theorem A. Let $G$ be a finite group whose order has prime factors $p_{1}, p_{2}, \cdots, p_{n}$. Let $L$ be the smallest term in the lower central series of $G$. The quotient $G / L$ is nilpotent and thus a direct product

$$
G / L \cong S_{1} \times S_{2} \times \cdots \times S_{n}
$$

with $S_{i}$ a (possibly trivial) $p_{i}$-group. For each $i$ let $P_{i}$ be some $p_{i}$-Sylow subgroup of G. Then

$$
\left|\gamma_{c+1}^{*}(G)\right| \leq \prod_{i=1}^{n} p_{i}^{\Lambda_{c}\left(S_{i}^{a b}\right)+\Psi_{c}\left(S_{i}\right)+\Theta_{c}\left(G, L, P_{i}\right)}
$$

The bound is attained, for instance, when $G$ is abelian. 
Note that if $G$ is perfect, then $\Lambda_{c}\left(S_{i}^{a b}\right)=0, \Psi_{c}\left(S_{i}\right)=0$ and $\Theta_{c}\left(G, L, P_{i}\right)=$ $d_{i}\left(2 \beta_{i}-d_{i}-1\right) / 2+\alpha_{i}$ where $p_{i}^{\beta_{i}}$ is the order of a $d_{i}$-generator $p_{i}$-Sylow subgroup $P_{i}$, and $p_{i}^{\alpha_{i}}$ is the order of the abelianisation $P_{i}^{a b}$. If, at the other extreme, $G$ is nilpotent, then we have $\Theta_{c}\left(G, L, P_{i}\right)=0$. If $G$ is abelian, then $\Theta_{c}\left(G, L, P_{i}\right)=0$ and $\Psi_{c}\left(S_{i}\right)=0$.

The bound in Theorem A can be sharpened by involving the relative Schur multiplier $\mathcal{M}(L, G)$ whose definition is recalled in Section 3. More precisely, in the definition of $\Theta_{c}(G, L, P)$ we can redefine $\omega=\mu+\beta^{\prime}$ where the $p$ th primary component of the abelian group $\mathcal{M}(L, G)$ has order $\left|\mathcal{M}(L, G)_{p}\right|=p^{\mu}$. For example, if $|L|$ is coprime to $|G| / \exp (L)$, then the relative multiplier is trivial (see Proposition $7($ ii) $)$ and we can take $\Theta_{c}\left(G, L, P_{i}\right)=\beta_{i}$ for $c \geq 1$.

Before stating result B let us recall that A. Lubotzky and A. Mann 23. defined a $p$-group $P$ to be powerful if: $p \geq 3$ and $[P, P] \subset P^{p}$; or $p=2$ and $[P, P] \subset P^{4}$ (where $P^{i}$ is the subgroup of $P$ generated by all $i$ th powers). In other words, $P$ is powerful if $p \geq 3$ and $P / P^{p}$ is abelian, or if $p=2$ and $P / P^{4}$ is abelian. They proved a number of results about powerful groups $P$, one of which states that the exponent $\exp \left(M^{(1)}(P)\right)$ of the Schur multiplier divides the exponent of $P$. We shall generalise this. Our generalisation implies, for instance, that $\exp \left(M^{(c)}(P)\right)$ divides $\exp (P)$ for all $c \geq 1$ and all $P$ in a certain class $C_{p}$ of $p$-groups; the class $C_{p}$ consists of those $p$-groups $P$ satisfying $\left[\left[P^{p^{i-1}}, P\right], P\right] \subset P^{p^{i}}$ for $1 \leq i \leq e$ where $\exp (P)=p^{e}$. It is shown in [23] that if $P$ is powerful, then $\left[P^{p^{i-1}}, P\right] \subset P^{p^{i}}$. Hence the class $C_{p}$ contains all powerful $p$-groups.

Given a normal subgroup $N \unlhd G$ of some group $G$, we say that the $\operatorname{pair}(N, G)$ has nilpotency class $k$ if $\gamma_{k+1}(N, G)=1$ and $\gamma_{k}(N, G) \neq 1$. For a real number $r$ we let $[r]$ denote the smallest integer $n$ such that $n \geq r$.

Let $N$ be a normal subgroup of a finite $p$-group $P$ and suppose that $N$ has exponent $p^{e}$. We define the integer

$$
\Omega(N, P)=\left[k_{1} / 2\right]+\left[k_{2} / 2\right]+\cdots+\left[k_{e} / 2\right]
$$

where $k_{j}$ denotes the nilpotency class of the pair $\left(N^{p^{j-1}} / N^{p^{j}}, P / N^{p^{j}}\right)$ for $1 \leq j \leq e$. For $N$ equal to the trivial group we set $\Omega(1, P)=0$. Note that $\Omega(N, P) \leq[k / 2] e$ where $k$ is the nilpotency class of $P$.

Theorem B. (i) Let $G$ be a finite group whose order has prime factors $p_{1}, p_{2}, \cdots$, $p_{n}$. Let $L$ be the smallest term in the lower central series of $G$. The quotient $G / L$ is thus a direct product

$$
G / L \cong S_{1} \times S_{2} \times \cdots \times S_{n}
$$

with $S_{i}$ a (possibly trivial) $p_{i}$-group. For each $i$ let $P_{i}$ be a $p_{i}$-Sylow subgroup of $G$. Suppose that $L \cap P_{i} /\left[L \cap P_{i}, P_{i}\right]$ has exponent $p_{i}^{n_{i}}$. Suppose that the $p_{i}$-primary component of $G^{a b}$ has exponent $p^{e_{i}}$ with $e_{i} \geq 0$, and set $m_{i}=\min \left(\Omega\left(L \cap P_{i}, P_{i}\right), e_{i}\right)$. Then, for each $c \geq 1$,

$$
\exp \left(\gamma_{c+1}^{*}(G)\right) \text { divides } \prod_{i=1}^{n} p_{i}^{\Omega\left(L \cap P_{i}, P_{i}\right)+\Omega\left(S_{i}, S_{i}\right)+n_{i}+(c-1) m_{i}} .
$$

The bound is attained if $G$ is abelian.

(ii) Suppose that a p-group $P$ satisfies $\left[\left[P^{p^{i-1}}, P\right], P\right] \subset P^{p^{i}}$ for all $1 \leq i \leq e$ where $p^{e}$ is the exponent of $P$. Then $\Omega(P, P)=e$. 
Note that, by inequality $(3), \exp \left(M^{(c)}(G)\right)$ divides $\exp \left(\gamma_{c+1}^{*}(G)\right)$ for any group $G$. Thus, for an arbitrary finite $p$-group $P$ of class $k$ and exponent $p^{e}$, Theorem B(i) implies that $\exp \left(M^{(c)}(P)\right)$ divides $p^{[k / 2] e}$; this sharpens the bound $\exp \left(M^{(c)}(P)\right) \leq$ $p^{(k-1) e}$ of Corollary 2.6 in [21 (for $c=1$ ) and Theorem 6 in [7] (for $c \geq 1$ ). Theorem $\mathrm{B}(\mathrm{ii})$ implies that $\exp \left(\gamma_{c+1}^{*}(P)\right)$ divides $\exp (P)$ if, for example, $P$ is a $p$-group with $P / P^{p}$ of nilpotency class 2 and $P^{p}$ contained in the second centre $Z_{2}(P)$.

The bound in Theorem $\mathrm{B}(\mathrm{i})$ can be sharpened by redefining $m_{i}$ to be $m_{i}=$ $\min \left(\epsilon_{i}, e_{i}\right)$ where $p_{i}^{\epsilon_{i}}$ and $p^{e_{i}}$ are the exponents of the $p_{i}$-primary components of $\mathcal{M}(L, G)$ and $G^{a b}$ respectively (cf. Proposition 7 in Section 3 ). The bound is clearly independent of $c$ if $G$ is finite nilpotent, or if $G$ is perfect. We do not know whether the bound can be made independent of $c$ for arbitrary finite groups.

For the statement of result $\mathrm{C}$ we let $D_{n}=\left\langle a, b \mid a^{2}=b^{n}=(a b)^{2}=1\right\rangle$ denote the dihedral group of order $2 m$, and $Q_{n}=\left\langle a, b \mid a^{2}=b^{n}=(a b)^{2}\right\rangle$ denote the quaternion group of order $4 n$. Recall $[3]$ that a $p$-group $E$ is said to be extra-special if its commutator subgroup $[E, E]$, its Frattini subgroup $\Phi(E)$, and its centre $Z_{1}(E)$ coincide and have order $p$. The extra-special groups have order $p^{2 k+1}$ for $k \geq 1$, with precisely two extra-special $p$-groups for each $k$ (see 3 ). We let $E(p, k)$ denote an arbitrary extra-special $p$-group of order $p^{2 k+1}$; we let $E(p, k)^{+}$and $E(p, k)^{-}$denote the extra-special $p$-groups of order $p^{2 k+1}$ and exponents $p$ and $p^{2}$ respectively. For $c \geq 1$ we have the following.

Theorem C. (i) For each $n \geq 2$ we have

$$
\gamma_{c+1}^{*}\left(D_{n}\right) \cong \begin{cases}C_{n} & \text { odd } n, \\ C_{n} \times\left(C_{2}\right)^{\chi_{c+1}(2)-1} & \text { even } n .\end{cases}
$$

The generator $b \in D_{n}$ acts trivially on $\gamma_{c+1}^{*}\left(D_{n}\right)$; the generator $a \in D_{n}$ acts trivially on elements of order two, and inverts the elements of the cyclic summand $C_{n}$.

(ii) For each $n \geq 2$ we have

$$
\gamma_{c+1}^{*}\left(Q_{n}\right) \cong \gamma_{c+1}^{*}\left(D_{n}\right) .
$$

The generators $a, b \in Q_{n}$ act as in (i).

(iii) For each $k \geq 2$ we have

$$
\gamma_{c+1}^{*}(E(p, k)) \cong\left(C_{p}\right)^{\chi_{c+1}(2 k)} .
$$

The group $E(p, k)$ acts trivially on $\gamma_{c+1}^{*}(E(p, k))$.

(iii)' For $p \geq 3$ and some $1 \leq r \leq 2^{c}$ we have

$$
\begin{gathered}
\gamma_{c+1}^{*}\left(E(p, 1)^{+}\right) \cong\left(C_{p}\right)^{\chi_{c+1}(2)+r}, \\
\gamma_{c+1}^{*}\left(E(p, 1)^{-}\right) \cong\left(C_{p}\right)^{\chi_{c+1}(2)} .
\end{gathered}
$$

Note that the corresponding Baer invariants $M^{(c)}(G)$ are easily obtained applying the formula $M^{(c)}(G)=\operatorname{ker}\left(\gamma_{c+1}^{*}(G) \rightarrow \gamma_{c+1}(G)\right)$ to the precise details of the isomorphisms given in the proof of Theorem $\mathrm{C}$. This extends the computations on dihedral 2-groups given in [16]. (We remark that there is a slip in the statement of the main theorem in [16]; the statement is correct for $\gamma_{c+1}^{*}\left(D_{2^{n}}\right)$ but incorrect for $M^{(c)}\left(D_{2^{n}}\right)$.)

The precise value of $r$ in Theorem $\mathrm{C}(\mathrm{iii})^{\prime}$ needs further investigation. The computer program listed in [12] yields the following results for the Burnside group $B(2,3)=E(3,1)^{+}$of exponent 3 on two generators. 


\begin{tabular}{lcl}
$c$ & $\gamma_{c+1}^{*}(B(2,3))$ & $r$ \\
\hline 1 & $\left(C_{3}\right)^{3}$ & 2 \\
2 & $\left(C_{3}\right)^{5}$ & 3 \\
3 & $\left(C_{3}\right)^{9}$ & 6 \\
4 & $\left(C_{3}\right)^{15}$ & 9 \\
5 & $\left(C_{3}\right)^{27}$ & 18
\end{tabular}

\section{Preliminaries}

The tensor product of nonabelian groups is a convenient setting for performing commutator calculations. Its functorial properties make it especially suited to the task of relating commutator calculations in a group to those in a homomorphic image of the group. We begin this section by recalling and developing relevant details on this tensor product. We then recall details on a Schur multiplier $\mathcal{M}(N, G)$ defined for pairs of groups. By a pair of groups $(N, G)$ we simply mean a group $G$ with normal subgroup $N$. The advantage of working with pairs is that any finite group $G$ can be expressed as an extension

$$
(L, G) \longleftrightarrow(G, G) \longrightarrow(G / L, G / L)
$$

of a 'perfect' pair $(L, G)$ by a 'nilpotent' pair $(G / L, G / L)$. Various simplifications apply when dealing with the Schur multiplier of perfect or nilpotent pairs. We end the section with some details on Baer invariants.

Suppose given two groups $G$ and $H$ which act on each other via group actions $G \times H \rightarrow H,(g, h) \mapsto{ }^{g} h$ and $H \times G \rightarrow G,(h, g) \mapsto{ }^{h} g$. Furthermore, suppose that each group acts on itself by conjugation, ${ }^{x} y=x y x^{-1}$. (In keeping with this notation, our convention for commutators is $[x, y]=x y x^{-1} y^{-1}$.) The tensor product $G \otimes H$ is defined [5], 4] to be the group generated by symbols $g \otimes h$ subject to the relations

$$
\begin{aligned}
& g g^{\prime} \otimes h=\left({ }^{g} g^{\prime} \otimes{ }^{g} h\right)(g \otimes h), \\
& g \otimes h h^{\prime}=(g \otimes h)\left({ }^{h} g \otimes{ }^{h} h^{\prime}\right),
\end{aligned}
$$

for $g, g^{\prime} \in G, h, h^{\prime} \in H$. The actions are said to be compatible if

$$
\left.{ }^{\left({ }^{g} h\right)} g^{\prime}={ }^{g}\left({ }^{h}\left(g^{-1} g^{\prime}\right)\right),{ }^{h} g\right) h^{\prime}={ }^{h}\left({ }^{g}\left(h^{-1} h^{\prime}\right)\right)
$$

for all $g, g^{\prime} \in G, h, h^{\prime} \in H$.

Proposition 1 ([5]). Suppose that $G$ and $H$ act compatibly on each other.

(i) For all $g, g^{\prime} \in G, h, h^{\prime} \in H$ the following identities hold in $G \otimes H$ :

$$
\begin{gathered}
g^{\prime} \otimes\left({ }^{g} h\right) h^{-1}=g^{\prime}(g \otimes h)(g \otimes h)^{-1}, \\
g\left({ }^{h} g^{-1}\right) \otimes h^{\prime}=(g \otimes h)^{h^{\prime}}(g \otimes h)^{-1}, \\
g\left({ }^{h} g^{-1}\right) \otimes\left({ }^{g^{\prime}} h^{\prime}\right) h^{\prime-1}=\left[g \otimes h, g^{\prime} \otimes h^{\prime}\right] .
\end{gathered}
$$

(ii) There is a homomorphism $\partial_{G}: G \otimes H \rightarrow G, g \otimes h \mapsto g^{h} g^{-1}$.

(iii) There is a 'diagonal' action of $G$ on $G \otimes H$ given by ${ }^{g^{\prime}}(g \otimes h)=\left(g^{\prime} g \otimes{ }^{g^{\prime}} h\right)$.

(iv) There is an isomorphism $G \otimes H \stackrel{\cong}{\rightarrow} H \otimes G, g \otimes h \mapsto h \otimes g$. 
(v) If ${ }^{g} h=h,{ }^{h} g=g$ for all $g \in G, h \in H$, then $G \otimes H \cong G^{a b} \otimes H^{a b}$, where the right-hand side of the isomorphism denotes the usual tensor product of abelian groups.

For each pair of groups $(N, G)$ we can form the tensor product $N \otimes G$ in which all actions are taken to be conjugation in $G$. Since conjugation yields compatible actions, there is a diagonal action of $G$ on $N \otimes G$. The tensor product $N \otimes G$ acts on G by conjugation in $G$ via the homomorphism $\partial_{N}: N \otimes G \rightarrow N$. We can thus construct the triple tensor product $(N \otimes G) \otimes G$. One readily checks that the construction preserves 'compatibility of actions', and that it can therefore be iterated to form the $(c+1)$-fold tensor product

$$
\bigotimes^{c+1}(N, G)=(\cdots((N \otimes G) \otimes G) \otimes \cdots \otimes G), \quad c \geq 1,
$$

involving $c$ copies of $G$ and one copy of $N$.

Proposition 2 ([13]). Let $G$ be a d-generator p-group with normal subgroup $N$. Suppose that $\left|\gamma_{i}(N, G)\right|=p^{m_{i}}$ for $i \geq 1, m_{i} \geq 0$. Then, for any $c \geq 1$, we have

$$
\left|\bigotimes^{c+1}(N, G)\right| \leq p^{m_{c} d+m_{c-1} d^{2}+\cdots+m_{1} d^{c}} .
$$

Lemma 3. Let $G_{3} \hookrightarrow G_{2} \rightarrow G_{1}, H_{3} \hookrightarrow H_{2} \rightarrow H_{1}$ be two short exact sequences of groups. Suppose that $G_{i}$ and $H_{i}$ act compatibly on one another for $1 \leq i \leq 3$, and that the homomorphisms preserve actions. Then there is an exact sequence of homomorphisms

$$
\left(G_{3} \otimes H_{2}\right) \overline{\times}\left(G_{2} \otimes H_{3}\right) \longrightarrow G_{2} \otimes H_{2} \longrightarrow G_{1} \otimes H_{1} \longrightarrow 1
$$

in which $\bar{x}$ denotes a semi-direct product whose details need not be specified.

Proof. The lemma is a routine adaption of Proposition 9 in [4].

Lemma 4. Let $N$ be a normal subgroup of $G$ for which the commutator $[n,[n, g]]$ is trivial for all $g \in G, n \in N$. In the tensor product $N \otimes G$, with $G$ and $H$ acting by conjugation, the following identity holds for all $g \in G, n \in N$ and all integers $t \geq 2$ :

$$
n^{t} \otimes g=(n \otimes g)^{t}\left(n \otimes[n, g]^{t(t-1) / 2}\right) .
$$

Proof. The case $N=G$ is proved in [2]. The proof of the more general case is analogous; it can also be derived directly using Proposition 1(i).

Recall that a pair $(N, G)$ is said to be nilpotent of class $k$ if $\gamma_{k+1}(N, G)=1$ and $\gamma_{k}(N, G) \neq 1$. Also recall that $[k / 2]$ denotes the smallest integer $n$ such that $n \geq k / 2$.

Proposition 5. Let $G$ be a group with normal subgroup $N$. Suppose that $N$ has prime-power exponent $p^{e}$ and that the pair $(N, G)$ has nilpotency class $\leq k$. Then, for any $c \geq 1$, we have

$$
\exp \left(\bigotimes^{c+1}(N, G)\right) \text { divides } p^{[k / 2] e}
$$


Proof. For $t=p^{e}$ the binomial coefficient $\left(\begin{array}{l}t \\ 2\end{array}\right)$ is divisible by $t$ when $p \geq 3$, and divisible by $t / 2$ when $p=2$. Thus Lemma 4 proves the proposition for $k=2, c=1$ (since for $p=2, t=p^{e}$ and $\gamma_{3}(N, G)=1$ the identity

$$
n \otimes[n, g]^{t / 2}=n \otimes\left[n^{t / 2}, g\right]
$$

holds for all $g \in G, n \in N$; but $\left[n^{t / 2}, g\right]=1$ because $n^{t / 2}$ has order at most 2.)

Let us now consider $k=2$ and some $c \geq 2$. Then

$$
\bigotimes^{c+1}(N, G)=\bigotimes^{c}(N \otimes G, G)
$$

and $N \otimes G$ acts trivially on $G$. The triviality of this action implies the identity

$$
\left(\cdots\left((n \otimes g)^{t} \otimes g_{1}\right) \otimes \cdots \otimes g_{c}\right)=\left(\cdots\left((n \otimes g) \otimes g_{1}\right) \otimes \cdots \otimes g_{c}\right)^{t}
$$

in $\bigotimes^{c+1}(N, G)$. Hence $\exp \left(\bigotimes^{c+1}(N, G)\right)$ divides $\exp (N \otimes G)$ and the proposition is proved for $k=2, c \geq 1$.

Suppose now that the proposition has been proved for some $c$ and all $k<k_{0}$. Suppose $\gamma_{k_{0}+1}(N, G)=1$. Lemma 3 implies an exact sequence

$$
\left(\gamma_{k_{0}-1}(N, G) \otimes G\right) \overline{\times}\left(N \otimes \gamma_{k_{0}-1}(N, G)\right) \rightarrow N \otimes G \rightarrow \frac{N}{\gamma_{k_{0}-1}(N, G)} \otimes \frac{G}{\gamma_{k_{0}-1}(N, G)} .
$$

Working in $N \otimes G$, the image of $\gamma_{k_{0}-1}(N, G) \otimes G$ contains the image of $N \otimes$ $\gamma_{k_{0}-1}(N, G)$ by virtue of the identity

$$
m \otimes[n, g]=([n, g] \otimes m)^{-1}
$$

which follows from Proposition 1(i) for all $g \in G, m, n \in N$. We thus have an exact sequence

$$
\gamma_{k_{0}-1}(N, G) \otimes G \rightarrow N \otimes G \rightarrow \frac{N}{\gamma_{k_{0}-1}(N, G)} \otimes \frac{G}{\gamma_{k_{0}-1}(N, G)} .
$$

By applying Lemma 3 to this sequence, and invoking a similar identity, we obtain the exact sequence

$$
\begin{aligned}
& \left(\gamma_{k_{0}-1}(N, G) \otimes G\right) \otimes G \rightarrow(N \otimes G) \otimes G \\
& \quad \rightarrow\left(\frac{N}{\gamma_{k_{0}-1}(N, G)} \otimes \frac{G}{\gamma_{k_{0}-1}(N, G)}\right) \otimes \frac{G}{\gamma_{k_{0}-1}(N, G)} .
\end{aligned}
$$

Repetition of the process yields an exact sequence

$$
\bigotimes^{c+1}\left(\gamma_{k_{0}-1}(N, G), G\right) \rightarrow \bigotimes^{c+1}(N, G) \rightarrow \bigotimes^{c+1}\left(\frac{N}{\gamma_{k_{0}-1}(N, G)}, \frac{G}{\gamma_{k_{0}-1}(N, G)}\right)
$$

from which we deduce that $\exp \left(\otimes^{c+1}(N, G)\right) \leq p^{\left[\left(k_{0}-2\right) / 2\right]} p^{e}=p^{\left[k_{0} / 2\right]}$. By induction, the proposition is proved for all $c, k \geq 1$.

Following J.-L.Loday [22] we say that a pair of groups $(N, G)$ is perfect if $N=$ $[N, G]$.

Proposition 6. Let $(N, G)$ be any perfect pair of groups and set

$$
M=\operatorname{ker}\left(\partial_{N}: N \otimes G \rightarrow N\right) .
$$

Then $M$ is abelian and, for each $c \geq 1$, there is an exact sequence

$$
\bigotimes^{c+1}\left(M, G^{a b}\right) \rightarrow \bigotimes^{c+2}(N, G) \rightarrow \bigotimes^{c+1}(N, G) \rightarrow 1
$$


where $\bigotimes^{c+1}\left(M, G^{a b}\right)$ is the usual iterated tensor product of abelian groups.

Proof. Let $H_{3} \hookrightarrow H_{2} \rightarrow H_{1}$ be a short exact sequence of groups, and let $G$ be a group such that $G$ and $H_{i}$ act compatibly on each other for $1 \leq i \leq 3$ with the homomorphisms preserving the actions. Then $H_{3}$ acts trivially on $G$ via $H_{2}$. Suppose that the action of $G$ on $H_{2}$ restricts to a trivial action of $G$ on $H_{3}$. Then Proposition 1(v) and Lemma 3 imply an exact sequence

$$
H_{3}^{a b} \otimes G^{a b} \rightarrow H_{2} \otimes G \rightarrow H_{1} \otimes G \rightarrow 1 \text {. }
$$

A perfect pair of groups $(N, G)$ gives rise to a short exact sequence $\operatorname{ker}\left(\partial_{N}\right) \hookrightarrow$ $N \otimes G \stackrel{\partial}{\rightarrow} N$. The identity

$$
{ }^{h}(n \otimes g)=([n, g] \otimes h)^{-1}(n \otimes g)
$$

which holds in $N \otimes G$ (see Proposition 1) for all $g, h \in G, n \in N$ implies that $G$ acts trivially on $\operatorname{ker}\left(\partial_{N}\right)$. So (5) implies an exact sequence

$$
M \otimes G^{a b} \rightarrow(N \otimes G) \otimes G \stackrel{\partial \otimes 1}{\longrightarrow} N \otimes G \rightarrow 1 .
$$

Note that the diagonal action of $G$ on $M \otimes G^{a b}$ is trivial, and hence $G$ acts trivially on $\operatorname{ker}(\partial \otimes 1)$. Thus a second application of (5) yields the exact sequence $\operatorname{ker}(\partial \otimes 1) \otimes G^{a b} \rightarrow \bigotimes^{4}(N, G) \rightarrow \bigotimes^{3}(N, G) \rightarrow 1$. From this we derive the exact sequence $\bigotimes^{3}\left(M, G^{a b}\right) \rightarrow \bigotimes^{4}(N, G) \rightarrow \bigotimes^{3}(N, G) \rightarrow 1$. The proposition follows from a repetition of this argument.

Given a pair of groups $(N, G)$ we denote by $\Delta(N, G)$ the subgroup of $N \otimes G$ generated by the elements $n \otimes n$ for $n \in N$. This is a normal subgroup and following [5] we define the exterior product

$$
N \wedge G=N \otimes G / \Delta(N, G) .
$$

The homomorphism $\partial_{N}: N \otimes G \rightarrow N$ clearly induces a homomorphism $\partial_{N}: N \wedge G \rightarrow$ $N$. The identity

$$
[g, n] \otimes\left[g^{\prime}, n^{\prime}\right]=\left[(g \otimes n),\left(g^{\prime} \otimes n^{\prime}\right)\right],
$$

of Proposition 1(i) implies an isomorphism $N \wedge G \cong N \otimes G$ in the case of perfect pairs.

Definition ([10]). The Schur multiplier of a pair of groups $(N, G)$ is the group $\mathcal{M}(N, G)$ defined by

$$
\mathcal{M}(N, G)=\operatorname{ker}\left(\partial_{N}: N \wedge G \rightarrow N\right) .
$$

If the pair is perfect, then, equivalently,

$$
\mathcal{M}(N, G)=\operatorname{ker}\left(\partial_{N}: N \otimes G \rightarrow N\right) .
$$

Proposition $7([10])$. Let $G$ be a finite group with normal subgroup $N \unlhd G$. $G$.

(i) Then $\mathcal{M}(N, G)$ is a finite abelian group with exponent e dividing the order of

(ii) Let $e^{\prime}$ denote the exponent of $N$. Then, in fact, ee divides the order of $G$ and $e$ divides the order of $N$.

(iii) Let $K$ be any subgroup of $G$ such that each $g \in G$ can be expressed (not necessarily uniquely) as a product $g=n k$ with $n \in N, k \in K$. Then $e^{2}$ divides $|N| \times|K|$. 
(iv) Suppose that $P$ is a $p$-Sylow subgroup of $G$. Let $\mathcal{M}(P \cap N, P)_{p}$ denote the p-component of the multiplier, and $\iota: \mathcal{M}(P \cap N, P) \rightarrow \mathcal{M}(N, G)$ the homomorphism induced by inclusion. Then

$$
\mathcal{M}(P \cap N, P) \cong \mathcal{M}(N, G)_{p} \oplus \operatorname{ker}(\iota) .
$$

Proposition 8. Let $G$ be a $\delta$-generator p-group with normal subgroup $N \unlhd G$ of order $|N|=p^{\beta}$. Suppose that $|N /(N \cap \Phi(G))|=p^{t}$ where $\Phi(G)=[G, G] G^{p}$. Then

$$
|N \wedge G| \leq p^{\delta \beta-\frac{t(t+1)}{2}} .
$$

Proof. Proposition 2 implies that $|N \otimes G| \leq p^{\delta \beta}$. There is a commutative diagram of group homomorphisms

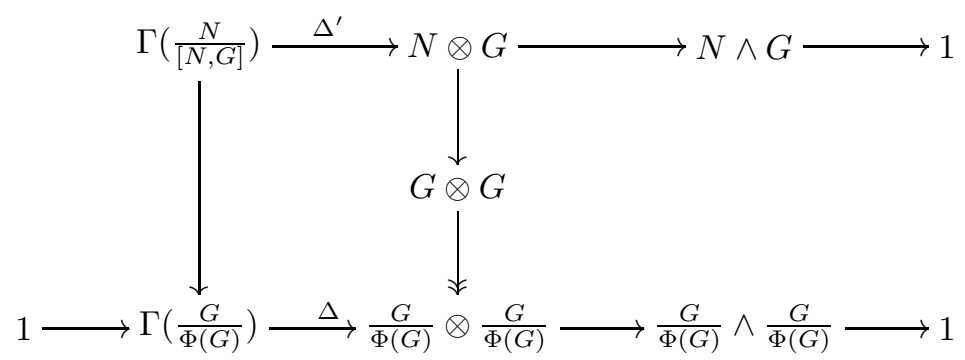

in which the rows (but not the columns) are exact [5. The abelian group $\Gamma(A)$, defined for any additive abelian group $A$, is J.H.C. Whitehead's universal quadratic construction; it is generated (as an abelian group) by symbols $\gamma(a)$ for $a \in A$ subject to the relations

$$
\begin{gathered}
\gamma(-a)=\gamma(a) \\
\gamma(a+b+c)+\gamma(a)+\gamma(b)+\gamma(c)=\gamma(a+b)+\gamma(a+c)+\gamma(b+c)
\end{gathered}
$$

for $a, b, c \in A$. The homomorphism $\Delta$ is defined on generators by $\Delta(\gamma(x))=x \otimes x$ for $x \in G / \Phi(G)$. The image of $\Gamma(G /[N, G])$ in $G / \Phi(G) \otimes G / \Phi(G)$ is an elementary abelian group of rank $t(t+1) / 2$. Hence the exactness of the top row implies

$$
|N \wedge G|=\frac{|N \otimes G|}{\left|\Delta^{\prime}(\Gamma(N /[N, G]))\right|} \leq \frac{p^{\delta \beta}}{p^{t(t+1) / 2}} .
$$

This proves the proposition.

Proposition 9. Let $N$ be a normal subgroup of a group $G$. If $N$ has exponent $p^{e}$, then

$$
\exp (N \wedge G) \text { divides } p^{\left[k_{1} / 2\right]+\left[k_{2} / 2\right]+\cdots+\left[k_{e} / 2\right]}
$$

where $k_{i}$ denotes the nilpotency class of the pair $\left(N^{p^{i-1}} / N^{p^{i}}, G / N^{p^{i}}\right)$ for $1 \leq i \leq e$.

Proof. Let $K, M$ be normal subgroups of $G$ with $K \leq M$. Using the identity $m \otimes k=(k \otimes m)^{-1}$ which holds in $M \wedge G$ for all $k \in K, m \in M$, one readily develops the short exact sequence

$$
K \wedge G \rightarrow M \wedge G \rightarrow M / K \wedge G / K \rightarrow 1
$$

from Lemma 3. Now (6) yields the exact sequences

$$
N^{p^{i}} \wedge G \rightarrow N^{p^{i-1}} \wedge G \rightarrow N^{p^{i-1}} / N^{p^{i}} \wedge G / N^{p^{i}} \rightarrow 1
$$


for $i \geq 1$. Hence

$$
\exp (N \wedge G) \leq \prod_{i=1}^{e} \exp \left(N^{p^{i-1}} / N^{p^{i}} \wedge G / N^{p^{i}}\right) .
$$

Proposition 5 implies $\exp \left(N^{p^{i-1}} / N^{p^{i}} \wedge G / N^{p^{i}}\right) \leq p^{\left[k_{i} / 2\right]}$.

Tensor products are related to Baer invariants by the following slight generalisation of a result of A.-S.T. Lue [24] (cf. [6]).

Proposition 10 (24]). For any group $G$ with normal subgroup $N \unlhd G$, and for $c \geq 1$, there is an exact sequence

$$
\bigotimes^{c+1}(N, G) \rightarrow \gamma_{c+1}^{*}(G) \rightarrow \gamma_{c+1}^{*}(G / N) \rightarrow 1
$$

It is convenient to set

$$
\bar{\gamma}_{c+1}^{*}(N, G)=\operatorname{ker}\left(\gamma_{c+1}^{*}(G) \rightarrow \gamma_{c+1}^{*}(G / N)\right) .
$$

Note that $\gamma_{c+1}^{*}(G)=\bar{\gamma}_{c+1}^{*}(G, G)$. (The bar is intended to suggest that $\bar{\gamma}_{c+1}^{*}(N, G)$ is a quotient of some functor $\gamma_{c+1}^{*}(N, G)$. For example, we can take $\gamma_{2}^{*}(N, G)=N \wedge G$ 6]. Proposition 9 and Proposition 11 could be subsumed under a single result concerning $\gamma_{c+1}^{*}(N, G)$.)

Proposition 11. Suppose that $N$ is a normal subgroup of a group $G$. If $N$ has exponent $p^{e}$, then

$$
\exp \left(\bar{\gamma}_{c+1}^{*}(N, G)\right) \text { divides } p^{\left[k_{1} / 2\right]+\left[k_{2} / 2\right]+\cdots+\left[k_{e} / 2\right]}
$$

where $k_{i}$ denotes the nilpotency class of the pair $\left(N^{p^{i-1}} / N^{p^{i}}, G / N^{p^{i}}\right)$ for $1 \leq i \leq e$. Proof. The proof is analogous to that of Proposition 9, but with (6) replaced by the exact sequence

$$
\bar{\gamma}_{c+1}^{*}(K, G) \rightarrow \bar{\gamma}_{c+1}^{*}(M, G) \rightarrow \bar{\gamma}_{c+1}^{*}(M / K, G / K) \rightarrow 1 .
$$

We leave the verification of the exactness of this (canonical) sequence to the reader.

The upper epicentral series of an arbitrary group $G$ was introduced in 6]. This is a family of characteristic subgroups $1=Z_{0}^{*}(G) \leq Z_{1}^{*}(G) \leq Z_{2}^{*}(G) \leq \cdots$ with various useful properties such as those listed in the next proposition. Part (i) of the following proposition can be taken as the definition of $Z_{c}^{*}(G)$.

Proposition $12(\underline{6})$. Let $c \geq 1$.

(i) $Z_{c}^{*}(G)$ is the smallest normal subgroup of $G$, contained in $Z_{c}(G)$, such that the quotient $G / Z_{c}^{*}(G)$ is isomorphic to $H / Z_{c} H$ for some group $H$.

(ii) $Z_{c+1}^{*}(G)$ contains $Z_{c}^{*}(G)$.

(iii) $Z_{c}^{*}(G)=1$ if and only if there exists an isomorphism $G \cong H / Z_{c} H$ for some group $H$.

(iv) Let $N$ be a normal subgroup of $G$. Then $N \leq Z_{c}^{*}(G)$ if and only if the quotient homomorphism $G \rightarrow G / N$ induces an isomorphism $\gamma_{c+1}^{*}(G) \stackrel{\cong}{\rightrightarrows} \gamma_{c+1}^{*}(G / N)$.

Let $A$ be a $d$-generator abelian group with generators $a_{1}, \cdots, a_{d}$. Let $A_{i}$ denote the cyclic subgroup of $A$ generated by $a_{i}$. Let $\mathcal{L}(d)$ denote the set of basic commutators on the $d$ symbols $a_{i}$. To each basic commutator $\lambda=\left[a_{i_{1}}, \cdots, a_{i_{k}}\right]$ of weight $k$ we associate the $k$-fold tensor product of abelian groups $T(\lambda)=A_{i_{1}} \otimes \cdots \otimes A_{i_{k}}$. 
Thus $T$ is a cyclic group of order equal to the highest common factor of the orders of the $A_{i_{j}}$. It is explained in [9] that the invariant $\gamma_{c+1}^{*}(A)$ is isomorphic to a direct sum of cyclic groups

$$
\gamma_{c+1}^{*}(A) \cong \bigoplus_{\lambda \in \mathcal{L}(d)} T(\lambda)
$$

The following proposition is an immediate corollary to this isomorphism. An alternative derivation of the proposition can be found in [28].

Proposition 13. Let $A$ be a direct product of cyclic groups

$$
A=\left(C_{n_{1}}\right)^{d_{1}} \times\left(C_{n_{2}}\right)^{d_{2}} \times \cdots \times\left(C_{n_{k}}\right)^{d_{k}}
$$

with each $n_{i}$ divisible by $n_{i+1}$. Then

$$
\gamma_{c+1}^{*}(A) \cong\left(C_{n_{1}}\right)^{\chi_{c+1}\left(d_{1}\right)} \times \prod_{j=2}^{k}\left(C_{n_{j}}\right)^{\left\{\chi_{c+1}\left(d_{1}+\cdots+d_{j}\right)-\chi_{c+1}\left(d_{1}+\cdots+d_{j-1}\right)\right\}} .
$$

Proposition $14(\underline{9})$. Let $G=S_{1} \times S_{2} \times \cdots \times S_{k}$ be a direct product of groups whose abelianisations $S_{i}^{a b}$ have finite, and mutually coprime, orders. Then, for each $c \geq 1$, there is an isomorphism

$$
\gamma_{c+1}^{*}(G) \cong \gamma_{c+1}^{*}\left(S_{1}\right) \times \cdots \times \gamma_{c+1}^{*}\left(S_{k}\right) .
$$

Proposition 15. Let $N$ be a nontrivial normal subgroup of a p-group $G$. Let $K$ denote the kernel of the canonical surjection $\gamma_{c+1}^{*}(G) \rightarrow \gamma_{c+1}^{*}(G / N)$. $G$.

(i) $K$ is nontrivial if and only if there exists some group $H$ for which $H / Z_{c} H \cong$

(ii) If $p \geq 3$ and $N \subset G^{p} \cap Z_{2} G$, or if $p=2$ and $N \subset G^{p^{2}} \cap Z_{2} G$, then $K$ is contained in the Frattini subgroup of $\gamma_{c+1}^{*}(G)$.

(iii) If $N$ is a proper subgroup of a cyclic normal subgroup in $G$, and if $N \subset Z_{2} G$, then $K$ is contained in the Frattini subgroup of $\gamma_{c+1}^{*}(G)$.

Proof. Proposition 12 implies (i).

Proposition 10 implies that $K$ is generated by the image of tensors of the form $\left(\cdots\left(\left(n \otimes g_{1}\right) \otimes g_{2}\right) \otimes \cdots \otimes g_{c}\right)$. The hypothesis of (ii) with Lemma 4 implies that the canonical image in $\bigotimes^{c+1}(G, G)$ of each such tensor lies in the subgroup $\otimes^{c+1}(G, G)^{p}$ generated by $p$ th powers of tensors. The hypothesis of (iii) implies that the image lies in the subgroup generated by $p$ th powers of tensors together with tensors of the form $\left(\cdots\left(\left(g \otimes g^{t}\right) \otimes g_{2}\right) \otimes \cdots \otimes g_{c}\right)$. In both cases $K$ lies in the Frattini subgroup of the $p$-group $\gamma_{c+1}^{*}(G)$.

\section{Proof of Theorem A}

Let $G, S_{i}, P_{i}, L$ be as in the statement of Theorem A. For each prime $p_{i}$ let $\bigotimes^{c+1}(L, G)_{p_{i}}$ denote some $p_{i}$-Sylow subgroup of $\bigotimes^{c+1}(L, G)$, and set

$$
\begin{gathered}
\Lambda_{c}^{i}=\log _{p_{i}}\left|\gamma_{c+1}^{*}\left(S_{i}^{a b}\right)\right|, \\
\Psi_{c}^{i}=\log _{p_{i}}\left|\bigotimes^{c+1}\left(\left[S_{i}, S_{i}\right], S_{i}\right)\right|, \\
\Theta_{c}^{i}=\log _{p_{i}}\left|\bigotimes^{c+1}(L, G)_{p_{i}}\right| .
\end{gathered}
$$


Propositions 10 and 14 imply exact sequences

$$
\begin{gathered}
\bigotimes(L, G) \rightarrow \gamma_{c+1}^{*}(G) \rightarrow \prod_{i=1}^{n} \gamma_{c+1}^{*}\left(S_{i}\right) \rightarrow 1, \\
\bigotimes\left(\left[S_{i}, S_{i}\right], S_{i}\right) \rightarrow \gamma_{c+1}^{*}\left(S_{i}\right) \rightarrow \gamma_{c+1}^{*}\left(S_{i}^{a b}\right) \rightarrow 1 .
\end{gathered}
$$

Hence

$$
\left|\gamma_{c+1}^{*}(G)\right| \leq \prod_{i=1}^{n} p_{i}^{\Lambda_{c}^{i}+\Psi_{c}^{i}+\Theta_{c}^{i}}
$$

To complete the proof we must find appropriate upper bounds $\Lambda_{c}\left(S_{i}^{a b}\right), \Psi_{c}\left(S_{i}\right)$, $\Theta_{c}\left(G, L, P_{i}\right)$ for $\Lambda_{c}^{i}, \Psi_{c}^{i}, \Theta_{c}^{i}$.

Proposition 13 furnishes the appropriate formula for $\Lambda_{c}\left(S_{i}^{a b}\right)$. Proposition 2 provides the appropriate formula for $\Psi_{c}\left(S_{i}\right)$. Suppose that $P_{i}$ is a $d_{i}$-generator group, that $P_{i} /\left(P_{i} \cap[G, G]\right)$ is a $\delta_{i}$-generator group, that $\left(L \cap P_{i}\right) /\left(L \cap \Phi\left(P_{i}\right)\right)$ has order $p^{t_{i}}$, that $L \cap P_{i}$ has order $p_{i}^{\beta_{i}}$, and that $\left[L \cap P_{i}, P_{i}\right]$ has order $p^{\beta_{i}^{\prime}}$. Set $M=\mathcal{M}(L, G)=\operatorname{ker}(L \otimes G \rightarrow L)$ and let $M_{p_{i}}$ denote the $p_{i}$-primary component of $M$. Since the pair $(L, G)$ is perfect, Proposition 6 implies

$$
\begin{aligned}
\left|\bigotimes^{c+1}(L, G)\right| \leq & \left|\bigotimes^{c}\left(M, G^{a b}\right)\right| \times\left|\bigotimes^{c-1}\left(M, G^{a b}\right)\right| \times \cdots \\
& \times \mid \bigotimes_{\left(M, G^{a b}\right)|\times| M|\times| L \mid}^{2}
\end{aligned}
$$

and thus

$$
\begin{aligned}
\left|\bigotimes^{c+1}(L, G)_{p_{i}}\right| \leq & \left|\bigotimes^{c}\left(M_{p_{i}}, G^{a b}\right)\right| \times\left|\bigotimes^{c-1}\left(M_{p_{i}}, G^{a b}\right)\right| \times \cdots \\
& \times\left|\bigotimes^{2}\left(M_{p_{i}}, G^{a b}\right)\right| \times\left|M_{p_{i}}\right| \times\left|L \cap P_{i}\right|
\end{aligned}
$$

Proposition $7\left(\right.$ iv) implies that $M_{p_{i}} \subset \mathcal{M}\left(L \cap P_{i}, P_{i}\right)$. Since $\left|L \cap P_{i}\right|=p_{i}^{\beta_{i}}$, Proposition 8 implies that

$$
\left|\mathcal{M}\left(L \cap P_{i}, P_{i}\right)\right| \times\left|\left[L \cap P_{i}, P_{i}\right]\right| \leq p_{i}^{d_{i} \beta_{i}-\frac{t_{i}\left(t_{i}+1\right)}{2}}
$$

Setting $\omega_{i}=d_{i} \beta_{i}-(1 / 2) t_{i}\left(t_{i}+1\right)$, we have

$$
\left|M_{p_{i}}\right| \leq\left|\mathcal{M}\left(L \cap P_{i}, P_{i}\right)\right| \leq p_{i}^{\omega_{i}-\beta_{i}^{\prime}}
$$

and hence

$$
\left|\bigotimes(L, G)_{p_{i}}\right| \leq p_{i}^{\left(\omega_{i}-\beta_{i}^{\prime}\right) \delta_{i}^{c}} p_{i}^{\left(\omega_{i}-\beta_{i}^{\prime}\right) \delta_{i}^{(c-1)}} \cdots p_{i}^{\left(\omega_{i}-\beta_{i}^{\prime}\right) \delta_{i}} p_{i}^{\left(\omega_{i}-\beta_{i}^{\prime}\right)} p_{i}^{\beta_{i}}
$$

This yields the appropriate formula for $\Theta_{c}\left(G, L, P_{i}\right)$. 


\section{Proof of Theorem B}

Let $G, S_{i}, P_{i}, L$ be as in the statement of Theorem B. Let $\bar{\gamma}_{c+1}^{*}(L, G)_{p_{i}}$ denote a $p_{i}$-Sylow subgroup of the group $\bar{\gamma}_{c+1}^{*}(L, G)$. The short exact sequence $\bar{\gamma}_{c+1}^{*}(L, G) \hookrightarrow$ $\gamma_{c+1}^{*}(G) \rightarrow \gamma_{c+1}^{*}(G / L)$ with Proposition 14 implies

$$
\exp \left(\gamma_{c+1}^{*}(G)\right) \leq \prod_{i=1}^{n} \exp \left(\bar{\gamma}_{c+1}^{*}(L, G)_{p_{i}}\right) \exp \left(\gamma_{c+1}^{*}\left(S_{i}\right)\right)
$$

We apply Propositions 6 and 10 and the surjection $\bigotimes^{c+1}(L, G) \rightarrow \bar{\gamma}_{c+1}^{*}(L, G)$ to obtain

$$
\exp \left(\gamma_{c+1}^{*}(G)\right) \leq \prod_{i=1}^{n} \exp \left(\left(\mathcal{M}(L, G) \otimes G^{a b}\right)_{p_{i}}\right)^{c-1} \exp \left((L \otimes G)_{p_{i}}\right) \exp \left(\gamma_{c+1}^{*}\left(S_{i}\right)\right)
$$

Proposition $7($ iv) yields

$$
\exp \left(\gamma_{c+1}^{*}(G)\right) \leq \prod_{i=1}^{n} \exp \left(\mathcal{M}\left(L \cap P_{i}, P_{i}\right) \otimes G^{a b}\right)^{c-1} \exp \left((L \otimes G)_{p_{i}}\right) \exp \left(\gamma_{c+1}^{*}\left(S_{i}\right)\right) \text {. }
$$

The exact sequence

$$
\left(L \cap P_{i}\right) \wedge P_{i} \rightarrow(L \otimes G)_{p_{i}} \rightarrow L \cap P_{i} /\left[L \cap P_{i}, P_{i}\right] \rightarrow 1
$$

is readily derived, and yields

$$
\begin{aligned}
\exp \left(\gamma_{c+1}^{*}(G)\right) \leq \prod_{i=1}^{n} & \exp \left(\mathcal{M}\left(L \cap P_{i}, P_{i}\right) \otimes G^{a b}\right)^{c-1} \exp \left(\left(L \cap P_{i}\right) \wedge P_{i}\right) \\
& \times \exp \left(\frac{L \cap P_{i}}{\left[L \cap P_{i}, P_{i}\right]}\right) \exp \left(\gamma_{c+1}^{*}\left(S_{i}\right)\right) .
\end{aligned}
$$

The bound of Theorem B(i) now follows from Propositions 9 and 11. (We take $N=G=S_{i}$ in Proposition 11.)

To prove Theorem $\mathrm{B}(\mathrm{ii})$ it suffices to note that the condition $\left[\left[P^{p^{i-1}}, P\right], P\right] \subset P^{p^{i}}$ is equivalent to saying that the pair $\left(P^{p^{i-1}} / P^{p^{i}}, P / P^{p^{i}}\right)$ has nilpotency class at most 2 .

\section{Proof of Theorem C}

Consider the dihedral group $D_{n}=\left\langle a, b \mid a^{2}=b^{n}=(a b)\right\rangle$ with $n=2^{r} m$ where $m \geq 1$ is odd. The smallest term of the lower central series of $D_{n}$ is $L=\gamma_{r}\left(D_{n}\right) \cong C_{m}$. Proposition 7 (ii) implies that the relative multiplier $\mathcal{M}(L, G)$ is trivial. Proposition 6 therefore implies an isomorphism $\otimes^{c+1}(L, G) \cong L \otimes G$ for all $c \geq 1$. So Proposition 10 yields a commutative diagram

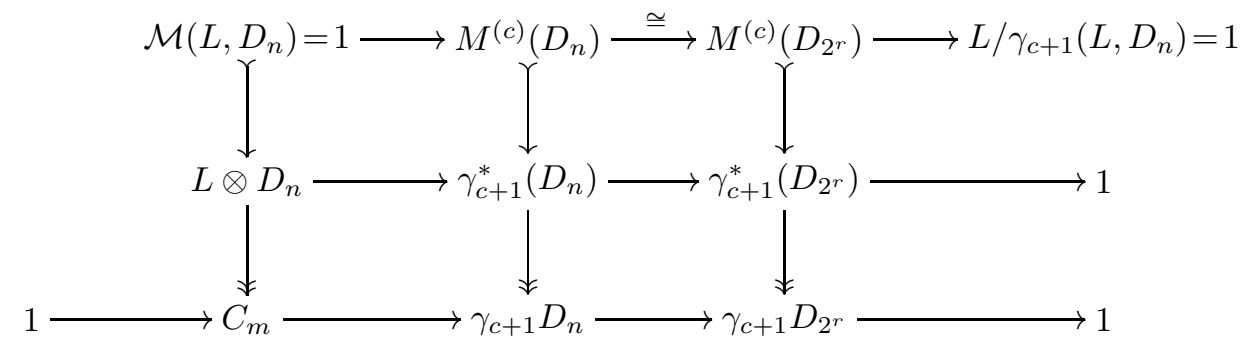


in which the rows are exact and the columns are short exact. From this, and the isomorphism $\gamma_{c+1} D_{n} \cong C_{m} \times \gamma_{c+1} D_{2^{r}}$, we derive the isomorphism

$$
\gamma_{c+1}^{*}\left(D_{n}\right) \cong C_{m} \times \gamma_{c+1}^{*}\left(D_{2^{r}}\right) .
$$

A description of $\gamma_{c+1}^{*}\left(D_{2^{r}}\right)$ is given in [16. Alternatively, the description can be re-obtained as follows. The central extensions $C_{2} \hookrightarrow D_{2^{r}} \rightarrow D_{2^{r-1}}$ and repeated applications of Proposition 15(i) imply that $\left|\gamma_{c+1}^{*}\left(D_{2^{r}}\right)\right| \geq\left|\gamma_{c+1}^{*}\left(C_{2} \times C_{2}\right)\right| \times 2^{r-1}$. These central extensions together with repeated applications of Proposition 15(iii) imply that $\gamma_{c+1}^{*}\left(D_{2^{r}}\right)$ has the same number of generators as $\gamma_{c+1}^{*}\left(C_{2} \times C_{2}\right)$, namely one generator for each basic commutator on two generators $a, b$. Theorem B implies that $\exp \left(\gamma_{c+1}^{*}\left(D_{2^{r}}\right)\right) \leq 2^{r}$. So, to obtain the isomorphism $\gamma_{c+1}^{*}\left(D_{2^{r}}\right) \cong C_{2^{r}} \oplus$ $\left(C_{2}\right)^{\chi_{c+1}(2)-1}$ it suffices to verify that at least all but one of the generators are of order 2 , and that the generators $a, b$ act as stated in the theorem. (Note that the invariant $\gamma_{c+1}^{*}(G)$ is abelian precisely when $\gamma_{c+1}(G)$ acts trivially on it.) This verification, which we leave to the reader, yields the desired description of $\gamma_{c+1}^{*}\left(D_{2^{r}}\right)$ and completes the proof of part (i) of the theorem.

To prove part (ii) we note that the quaternion group $Q_{n}$ is not of the form $H / Z(H)$ for any group $H$ with centre $Z(H)$ [3]. Proposition 12(ii)(iii) thus implies that the $c$ th term $Z_{c}^{*}\left(Q_{n}\right)$ of the upper epicentral series of $Q_{n}$ contains the centre $Z\left(Q_{n}\right) \cong C_{2}$. Since $Q_{n} / Z\left(Q_{n}\right) \cong D_{n}$, the isomorphism $\gamma_{c+1}^{*}\left(Q_{n}\right) \cong \gamma_{c+1}^{*}\left(D_{n}\right)$ follows from Proposition 12 (iv).

To prove part (iii) we note that an extraspecial group $E(p, k), k \geq 2$, is not of the form $H / Z(H)$ for any group $H$ with centre $Z(H)$ 3. Arguing as in the previous paragraph, we see that $\gamma_{c+1}^{*}(E(p, k)) \cong \gamma_{c+1}^{*}(E(p, k) / Z(E(p, k))) \cong \gamma_{c+1}^{*}\left(C_{p} \times C_{p}\right)$. Proposition 13 completes the proof of part (iii). This argument also holds for $E(p, 1)^{-}, p \geq 3$.

To obtain our partial description of $\gamma_{c+1}^{*}\left(E(p, 1)^{+}\right), p \geq 3$, we first remark that $Z_{1}^{*}(G)$ is trivial for the group $G=E(p, 1)^{+}$[3]. Letting $Z=Z(G)$ denote the centre of this group, Proposition 10 and Proposition 1(v) yield an exact sequence $\otimes^{c+1}(Z, G)=Z \otimes G^{a b} \otimes \cdots \otimes G^{a b} \rightarrow \gamma_{c+1}^{*}(G) \stackrel{\phi}{\longrightarrow} \gamma_{c+1}^{*}\left(C_{p} \times C_{p}\right) \rightarrow 1$. The group $\otimes^{c+1}(Z, G)$ is elementary abelian of rank $2^{c}$, and Proposition 12(iv) implies that $\phi$ has non-trivial kernel. Theorem B implies that $\exp \left(\gamma_{c+1}^{*}(G)\right)=p$. The commutator subgroup $[G, G]=Z$ acts trivially on $\gamma_{c+1}^{*}(G)$, and so $\gamma_{c+1}^{*}(G)$ is abelian. Hence $\gamma_{c+1}^{*}(G)$ is elementary abelian of rank at most $\chi_{c+1}(2)+2^{c}$, and at least $\chi_{c+1}(2)+1$.

\section{ACKNOWLEDGEMENTS}

This research was carried out at the Max Planck Institut für Mathematik, Bonn. I would like to thank the Institute for its very generous hospitality.

\section{REFERENCES}

[1] Baer, R.: Representations of groups as quotient groups, I, II, and III. Trans. Amer. Math. Soc. 58, 295-419 (1945) MR 7:371h; MR 7:372a MR 7:372b

[2] Bacon, M.R. Kappe, L.-C.: The nonabelian tensor square of a 2-generator $p$-group of class 2. Archiv Math. 61, 508-516 (1993) MR 95h:20041

[3] Beyl, F.R., Tappe, J.: Group extensions, representations, and the Schur multiplicator. Lecture Notes in Mathematics 958, Springer (1982) MR 84f:20002

[4] Brown, R. Johnson, D.L. Robertson, E.F.: Some computations of nonabelian tensor products of groups. J. Algebra 111, 177-202 (1987) MR 88m:20071

[5] Brown, R. Loday, J.-L.: Van Kampen theorems for diagrams of spaces. Topology 26, 311-335 (1987) MR 88m:55008 
[6] Burns, J., Ellis, G.: On the nilpotent multipliers of a group. Math. Z. 226, 405-428 (1997) MR 98h:20050

[7] Burns, J., Ellis, G.: Inequalities for Baer invariants of finite groups. Canad. Math. Bull. 41 385-391 (1998) MR 99i:20028

[8] Burns, J., Ellis, G., MacHale, D., Ó'Murchú, P., Sheey, R., Wiegold, J.: Lower central series of groups with small upper central factors. Proc. Roy. Irish Acad. Sect. A 97, 113-122 (1997) MR 99g:20061

[9] Ellis, G.: On groups with a finite nilpotent upper central quotient. Archiv Math. 69, 1-8 (1997) MR 99a:20033

[10] Ellis, G.: The Schur multiplier of a pair of groups. Appl. Categ. Structures 6, no. 3, 355-371 (1998) MR 99h:20086

[11] Ellis, G.: A bound for the derived and Frattini subgroups of a prime-power group. Proc. Amer. Math. Soc. 126, No. 9, 2513-2523 (1998) MR 98k:20028

[12] Ellis, G.: On the computation of certain homotopical functors. LMS J. Comput. Math. 1, 25-41 (1998) MR 99f:55002

[13] Ellis, G., McDermott, A.: Tensor products of prime-power groups. J. Pure Appl. Algebra 132, no. 2, 119-128 (1998) MR 99g:20049

[14] Frölich, A.: Baer invariants of algebras. Trans. Amer. Math. Soc. 109, 221-244 (1962)

[15] Gaschütz, W., Neubüser, J., and Yen, Ti.: Über den Multiplikator von $p$-Gruppen. Math. Z., 100, 93-96 (1967) MR 36:272

[16] Gupta, N.D., Moghaddam, M.R.R.: Higher Schur-multiplicators of nilpotent dihedral groups. C.R. Math. Rep. Acad. Sci. Canada, Vol. 14, no. 5, 225-230 (1993) MR 94f:20070

[17] Green, J.A.: On the number of automorphisms of a finite group. Proc. Royal Soc. A 237, 574-581 (1956) MR 18:464c

[18] Hall, P.: The Edmonton notes on nilpotent groups. Queen Mary College Mathematics Notes. (1969) MR 44:316

[19] Jones, M.R.: Multiplicators of p-groups. Math. Z. 127, 165-166 (1972) MR 47:6851

[20] Jones, M.R.: Some inequalities for the multiplicator of a finite group. Proc. Amer. Math. Soc. 39, 450-456 (1973) MR 47:3524

[21] Jones, M.R.: Some inequalities for the multiplicator of a finite group II. Proc. Amer. Math. Soc. 45, 167-172 (1974) MR 50:4741

[22] Loday, J.-L.: Cohomologie et groupes de Steinberg relatifs. J. Algebra 54, 178-202 (1978) MR 80b:18013

[23] Lubotzky, A., Mann, A.: Powerful p-groups I. Finite groups. J. Algebra 105, no. 2, 484-505 (1987) MR 88f:20045

[24] Lue, A.-S.T.: The Ganea map for nilpotent groups. J. London Math. Soc. 14, 309-312 (1976) MR 55:3110

[25] MacDonald, J.L.: Group derived functors. J. Algebra 10, 448-477 (1968) MR 40:205

[26] MacHale, D, Ó’Murchú, P.: Commutator subgroups of groups with small central factor groups. Proc. Roy. Irish Acad. Sect. A 93, no. 1, 123-129 (1993) MR 95b:20058

[27] Magnus, W., Karrass, A., Solitar, D.: Combinatorial Group Theory. New York (1976) MR 54:10423

[28] Mashayekhy, B., Moghaddam, M.R.R.: Higher Schur-multiplicator of a finite abelian group. Algebra Colloquim 4:3, 317-322 (1997) MR 2000a:20079]

[29] Wiegold, J.: Multiplicators and groups with finite central factor groups. Math. Z. 89, 345-347 (1965) MR 31:3510

[30] Wiegold, J.: Commutator subgroups of finite p-groups. J. Australian Math. Soc., 10, 480-484 (1969) MR 41:3606

Max-Planck-Institut für Mathematik, Gottfried-Claren-Strasse 26, Bonn, Germany Current address: Department of Mathematics, National University of Ireland, Galway, Ireland E-mail address: graham.ellis@nuigalway.ie 